\title{
Reconstruction of frontal defects with calvarial grafts*
}

\author{
Stamatia Dova, Petros D. Karkos, Jannis Constantinidis \\ Department of Otolaryngology-Head and Neck Surgery, AHEPA Hospital, Aristotle University of Thessaloniki, Greece
}

Rhinology 56: 3, 297-302, 2018

https://doi.org/10.4193/Rhin17.185

*Received for publication:

August 17, 2017

Accepted: February 25, 2018

Background: There are many options for the reconstruction of frontal sinus wall defect resulting from injury, neoplastic lesions and aesthetic deformities. These mainly include autogenous grafts, allogenous grafts, and alloplastic materials. The aim of the present study is to report the advantages of using autogenous calvarial bone grafts over other reconstruction techniques, in the reconstruction of frontal defects.

Methods: We describe 16 consecutive cases of anterior frontal sinus defects between 2004 and 2015, in which calvarial bone grafts were used. Medical records were retrospectively analysed to evaluate graft survival.

Results: Bone defects were caused by injury (3), aesthetic deformities (2), external frontal sinus surgeries (2), tumours (6: three osteomas, two fibrous dysplasias, one squamous cell carcinoma), and osteomyelitis (3). There were no significant complications during harvesting, and morbidity was minimal. Furthermore, at one and five-year follow-up, no graft resorption or rejection was noted, and cosmetic results were satisfactory.

Conclusions: Our results indicate that calvarial bone graft is an appropriate material to use in anterior frontal sinus reconstruction.

Key words: calvarial, defect, frontal, graft, reconstruction

\section{Introduction}

The skull, by embryological origin, is divided into three distinct parts: the chondrocranium, the desmocranium and the visceral portion. The importance of the above embryological differentiation lies in the fact that, according to studies, grafts of membranous bone origin (calvarium) are superior to those of endochondral formation (iliac crest), in respect to resorption (1). Calvarium was first used in bone grafting by Müller and König in $1890^{(2,3)}$. It consists of the internal and external tables of compact bones (inner and outer cortex), separated by a spongy cancellous layer (diploe) lying between them ${ }^{(4)}$. Defects in the frontal region usually occur due to injuries, resection of tumours (iatrogenic), congenital lesions, and infections ${ }^{(5)}$. Reconstruction of the bony forehead aims to restore the normal contour and aesthetics. The most difficult issue in these cases is selecting the optimal material. An ideal reconstruction material should be bio-combatible, cost-effective, malleable and stable. Alloplastic materials act as foreign bodies and are prone to infections ${ }^{(5)}$. The aim of our study is to assess calvarial bone graft as a possible suitable material for bony forehead reconstruction.

\section{Materials and methods}

Following the approval of the Institutional Ethics Review Board, we retrospectively reviewed the medical records of patients who had undergone calvarial bone grafting by the senior author (J.C.) between 2004 and 2015, for defects of the anterior frontal sinus wall. The principles of Helsinki declaration were followed. Records were evaluated for patient demographics, causes of defects, presenting symptoms, imaging modalities, surgical technique, and short and long-term follow-up results.

\section{Surgical technique}

Written consent was obtained preoperatively, and all patients were informed of the possibility of a minor flattening at the donor site. The initial part of the operation is based on the principles of the osteoplastic frontal flap approach through a coronal 


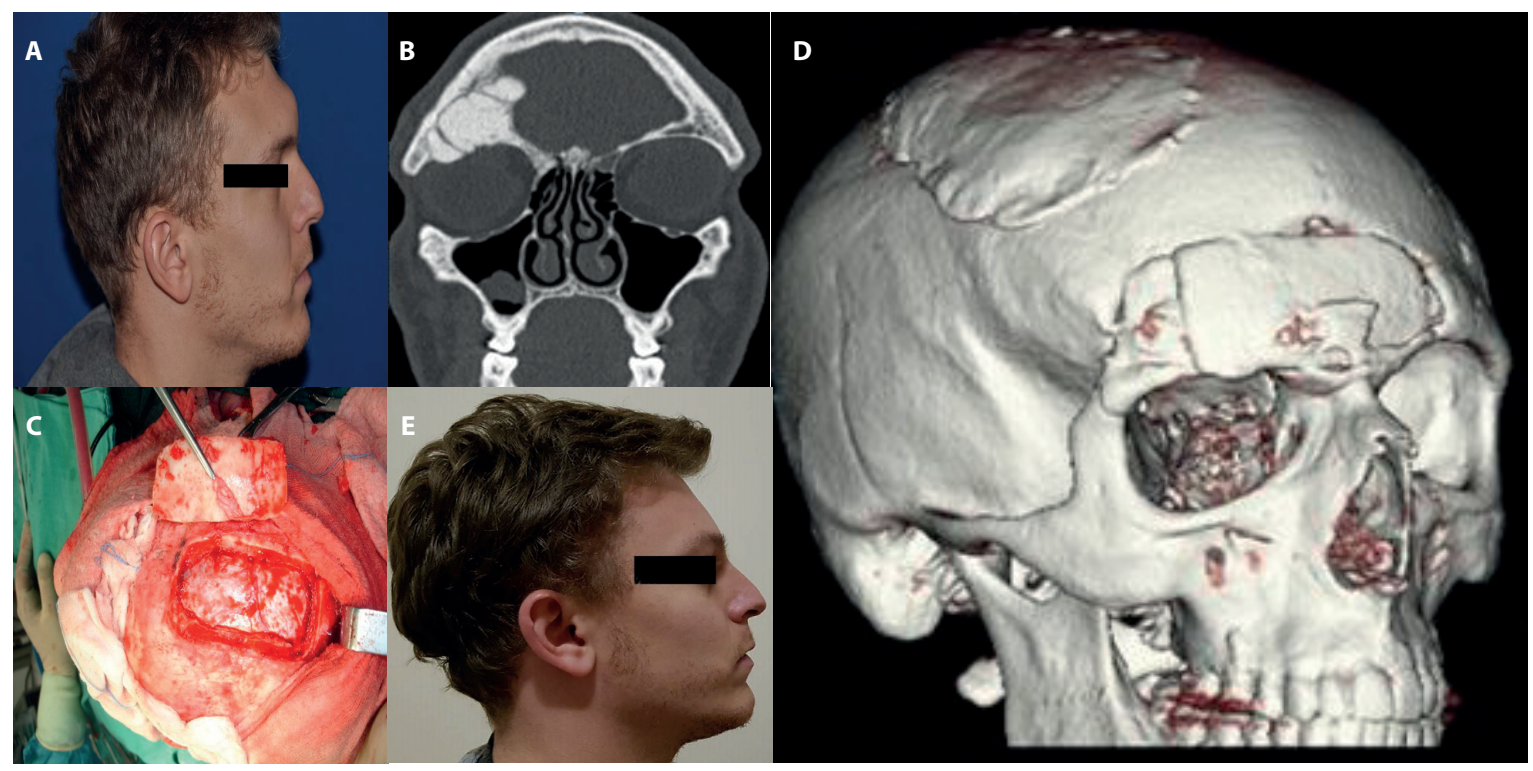

Figure 1 A-E. Giant frontal sinus osteoma with intracranial and intraorbital extension in a 30-year-old man. A. Preoperative lateral view of the patient showing forehead protuberance. B. Preoperative CT scan (coronal bone window). C. Harvesting of the bicortical calvarial bone graft following tumour resection. D. Postoperative 3D reconstruction CT scan. E. Postoperative view of the patient at four-year follow-up, without any residual cosmetic deformity.

incision ${ }^{(6)}$. We didn't shave hair, especially in women, in order to avoid a long-term stigmatisation caused by an unacceptable scar. In bald men, the incision was replaced by a horizontal one, concealed into a forehead furrow line or performed further back. It is worth noting that, prior to reconstruction, residual frontal sinus disease must be eradicated, and frontal sinus drainage into the nose (type III drainage) or sufficient exclusion of paranasal sinuses must be performed (obliteration procedure). In three cases of bone infection (osteomyelitis), debridement of the diseased bone and tissue removal of healthy tissue with safety margins was initially required.

In order to create an optimal fit to the recipient site, a thermoplast template was created intraoperatively. The donor site in all cases was the parietal region, approximately $2 \mathrm{~cm}$ lateral to the midline, in order to protect the superior sagittal sinus. Three types of calvarial bone grafts can be harvested. These include full-thickness outer cortex grafts, partial-thickness outer cortex grafts, and bicortical or inner cortex grafts. The most frequent type involves removal of the outer cortex in its entirety ${ }^{(4)}$. In 14 cases we used full-thickness outer cortex grafts and in two cases bicortical grafts. The graft was outlined using a small drill or a cutting burr, and the elevation of the outer cortex was performed using an oscillating saw or chisels, by splitting along the diploic space (full thickness outer cortex graft). In cases of very thin diploe or cranium, a bicortical graft (complete calvarium graft) was performed. The graft was outlined and the bone was drilled up to the dura, harvested, and elevated from the dura with a punch. Alternatively it can be harvested via a craniotomy, in collaboration with a neurosurgeon.

Following the fitting of the graft to the recipient site, rigid fixation was achieved by using miniplates or microplates and/or non-absorbable sutures. Refinement was carried out using a diamond burr. In order to avoid irregularities of the recipient site, a temporalis fascia graft was used to cover the margins, especially in cases of thin scalp. The temporalis fascia is easily accessible through the same operative field. Apparent crests or ridges are rare postoperatively when full thickness outer cortex grafts are used. However, deformities of the donor site can be avoided via restoration with alloplastic materials such as Palacos, Medpor or hydroxyapatite. There was a need for such reconstruction in two cases of full thickness outer cortex grafts and in two cases of bicortical grafts. Reconstruction of the donor site is mandatory in cases of bicortical graft harvesting not only for aesthetic reasons, but also to protect the brain from external traumas.

The surgical wound was closed with staples, and a head bandage was applied exerting light pressure. The patient was also instructed to wear a tennis headband for 6 weeks postoperatively. On average, patients were discharged on the 5 th postoperative day.

\section{Results}

The causes of the defects were 3 severe injuries, 2 aesthetic deformities, 2 external frontal sinus surgeries (Riedel procedure), 6 tumours ( 3 osteomas, 2 fibrous dysplasias, 1 squamous cell carcinoma), and 3 cases of osteomyelitis. Patient age ranged from 27 to 65 years. A CT scan with 3D reconstruction and an 
Table 1. Reconstruction of the forehead with calvarial grafts $(n=16)$. Patient characteristics.

\begin{tabular}{|c|c|c|c|c|c|}
\hline Patient, age & Indication & Graft material & $\begin{array}{l}\text { Frontal sinus } \\
\text { management }\end{array}$ & $\begin{array}{l}\text { Donor site } \\
\text { reconstruction }\end{array}$ & Recent Follow- up \\
\hline$M, 30 y$ & Osteoma & BCG & Obliteration & Palacos & $4 y$ \\
\hline$M, 42 y$ & Osteoma & FTOG & Type III drainage & no & $4 y$ \\
\hline$F, 59 y$ & Osteoma & FTOG & Type III drainage & no & $4 y$ \\
\hline$F, 27 y$ & Fibrous dysplasia & FTOG & Obliteration & no & $5 y$ \\
\hline$M, 48 y$ & Fibrous dysplasia & BCG & Obliteration & Palacos & $5 y$ \\
\hline F, 55y & Primary SCC & FTOG & Obliteration & no & $9 y$ \\
\hline$F, 42 y$ & Riedel procedure & FTOG & Obliteration & no & $5 y$ \\
\hline$M, 65 y$ & Riedel procedure & FTOG & Obliteration & no & $2 y$ \\
\hline F, 55y & Osteomyelitis & FTOG & Obliteration & Palacos & $5 y$ \\
\hline$M, 32 y$ & Osteomyelitis & FTOG & Obliteration & no & $5 y$ \\
\hline$F, 61 y$ & Osteomyelitis & FTOG & Obliteration & no & $5 y$ \\
\hline M, 28y & Injury & FTOG & Type III drainage & no & $5 y$ \\
\hline M, 39y & Injury & FTOG & Type III drainage & no & $5 y$ \\
\hline$F, 52 y$ & Injury & FTOG & Type III drainage & no & $5 y$ \\
\hline M, 34y & Aesthetic deformity & FTOG & Type III drainage & no & $4 y$ \\
\hline$F, 28 y$ & Aesthetic deformity & FTOG & Type III drainage & Palacos & $4 y$ \\
\hline
\end{tabular}

M: male, F: female, BCG: bicortical calvarial bone graft, FTOG: full-thickness outer cortex bone graft, SCC: squamous cell carcinoma

MRI scan were arranged preoperatively, and at 3 and 12 months postoperatively (Table 1). Three representative case reports are described in the legends of Figures 1 to 3.

We performed type III drainage in all cases of injury and aesthetic deformity, and in two cases of osteoma. At follow-up, patients who underwent type III drainage had a patent neoostium, without any restenosis or need for revision surgery. Two patients had a history of a previous operation. For all nine patients with tumour and injury, reconstruction was accomplished during the same operation. On the contrary, in the cases of osteomyelitis, reconstruction was performed at a second stage. In all cases, at 3 and 12- month follow-up, the obtained CT scan demonstrated stability of the shape of the graft and absence of bone resorption. Additionally, at one-year follow-up there was no graft rejection, dislocation or inflammation. During the follow-up period between 2 and 9 years, the calvarial bone grafts remained stable in size and shape. In all 16 cases there were no intraoperative complications during harvesting. Expected intraoperative bleeding, arising from transcortical emissary veins, subcortical vessels and arachnoid plexuses, within the cortical portion of the calvarium, was successfully controlled using bone wax, hemostatic matrix (Floseal) or a diamond drill. Finally, long-term cosmetic results were satisfactory.

\section{Discussion}

Autogenous grafts, allogenic grafts and alloplastic materials have all been used in the reconstruction of the anterior frontal sinus wall ${ }^{(7)}$. Autogenous grafts comprise of free bone grafts, free cartilage grafts and pedicled myo-osseous flaps. Autogenous grafts can be compact (rib, scapula), cancellous (iliac crest), or split bone grafts. Compared to split bone grafts, other bone and cartilage grafts have the disadvantage of an additional operative field and a high rate of resorption ${ }^{(8)}$, while myo-osseous flaps require a difficult surgical technique. Allogenic grafts, which consist of preserved cartilage or bone, are small with high resorption rate ${ }^{(8)}$. Alloplastic materials may consist of metal (titanium plates or mesh), plastic (polumethylmethacrylate), ceramic (hydroxyapatite or carbonated apatite), and biocement (bioverit) ${ }^{(7)}$. They are usually expensive materials, with plastic grafts presenting the disadvantage of inflammation ${ }^{(5)}$, and ceramic grafts a higher rate of resorption and migration ${ }^{(9,10)}$.

Calvarium is cost-effective, with minimum morbidity and good long-term cosmetic results. Danger areas that should be avoided during graft harvesting include the midline which the sagittal sinus traverses, the region inferior to the temporal line where the skull is thin, and suture regions (sagittal, coronal, lambdoid, and squamosal), due to the fusion between cortices at these sites ${ }^{(4)}$. Before harvesting, requirements of the recipient site should 


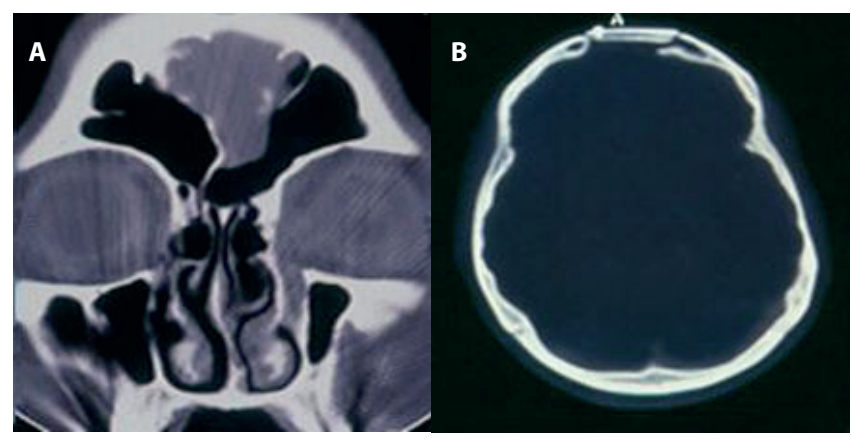

Figure 2 A-B. Primary squamous cell carcinoma of the frontal sinus in a 55-year-old woman. A. Computed tomography scan (coronal view). B. Postoperative CT scan at one-year follow-up after resection of the tumour and obliteration of the frontal sinus. Neither recurrence of the tumour nor resorption of the outer cortex graft were noted (axial view).

be taken under consideration. Strips from the temporoparietal region are preferred for curved grafts, while the occipitoparietal region is the most suitable for straight grafts ${ }^{(4)}$. Partial thickness outer cortex grafts should not be longer than $6 \mathrm{~cm}$ and wider than $2 \mathrm{~cm}$, as there is a higher risk of fracture and intracranial exposure during harvesting ${ }^{(4)}$.

The main advantages of calvarial split bone grafts over other grafts are better graft survival, harvesting through the same operative field, minimal pain and discomfort for the patient, the predictable contour and the possibility of use in contaminated spaces (infections in paranasal sinuses or in cases of injury) ${ }^{(11,12)}$. A predominant advantage of these grafts is the potential use in irradiated regions and under irradiated skin. The blood supply of the recipient bed is excellent allowing successful bone transplantation. Compared to alloplastic materials, autogenous calvarial bone grafts present better mechanical, biological, and immunological characteristics ${ }^{(13)}$. Nevertheless, titanium mesh implant is an alternative to calvarial grafts, especially in cases with large cranial defects, as it is inert, malleable and has the ability to osseointegrate ${ }^{(14)}$. Additionally, advanced 3D printing techniques have permitted the manufacturing of porous titanium implants, customised to the individual needs of patients with such defects, without leaving any dead space, but at a rather high cost ${ }^{(15)}$.

Complications occur at a low rate, and are divided in immediate such as donor site deformity, fracture of the graft, intracerebral hematoma ${ }^{(4,7,16,17)}$ and delayed complications, mainly infections (17). In long-term follow-up there were no complications, such as infection or resorption. In contrast, we have seen patients with such problems, following implantation of alloplastic materials. In his retrospective analysis of 98 patients, who underwent cranioplasty with methylmethacrylate, Marchac reports that 89 of them had excellent results. Infection and implant removal rate was very high, when nasal or frontal sinus tissue was in direct contact with the implant during the operative procedure ${ }^{(18)}$. Friedman evaluated 39 patients with acute traumatic or acute surgically induced defects of the frontal sinus and anterior craniofacial skeleton, which were reconstructed with hydroxyapatite cement. With no history of significant sinus mucoperiosteal disease, the success rate was $93 \%$. However, patients with a history of sinus mucoperiosteal disease presented a higher risk of complications ${ }^{(9)}$.

Type III drainage prior to grafting was performed in all cases of injury or aesthetic deformity, and in two cases of osteoma. Main indications for Draf III procedure include difficult revision surgery, revision surgery for chronic rhinosinusitis and patients with risk factors, such as the ASA triad, ciliary dyskinesia syndrome, generalised polyposis and benign tumours with the main portion medial to the lamina papyracea, and certain malignant
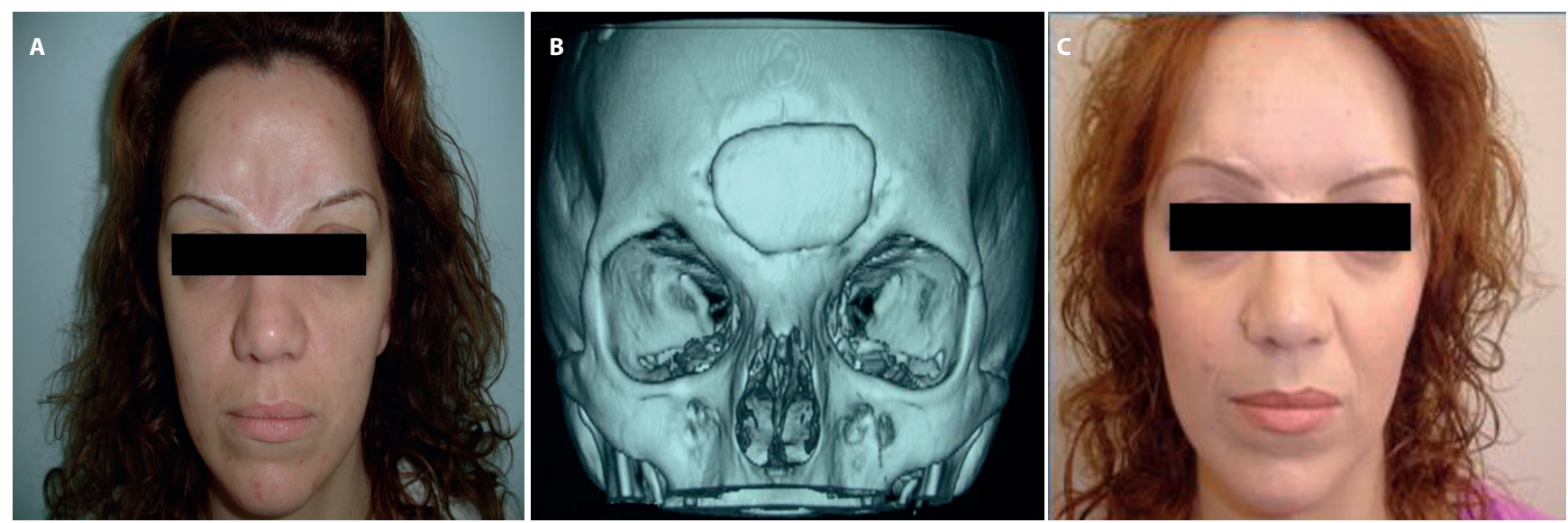

Figure 3 A-C. Forehead cosmetic deformity following a previous Riedel procedure in a 42-year-old woman. A. Preoperative photo of the patient. B. Postoperative 3D reconstruction CT scan at one-year follow-up. Stability of the outer cortex bone graft. C. Postoperative photo of the patient at 5-year follow-up, with favourable aesthetic outcome. 
Table 2. Use of calvarial grafts in craniofacial surgery with favourable results (literature data)

\begin{tabular}{|llc|}
\hline Study & \multicolumn{1}{c}{ Cause of defect } & Follow-up \\
\hline Chang et al. 1997 & frontal sinus osteoma (1) & $3.5 \mathrm{y}$ \\
\hline Liu et al. 2001 & $\begin{array}{l}\text { aneurysmal bone cyst of frontal } \\
\text { sinus (1) }\end{array}$ & $1 \mathrm{y}$ \\
\hline Hendus et al. 2005 & $\begin{array}{l}\text { tumours (5), injuries (6), osteo- } \\
\text { myelitis (4) }\end{array}$ & $2-8 \mathrm{y}$ \\
\hline Gil et al. 2007 & $\begin{array}{l}\text { anterior skull base tumors (10) } \\
\text { Lubbers et al. 2011 }\end{array}$ & $\begin{array}{l}\text { osteoblastoma of } \\
\text { frontal,temporal skull (1) }\end{array}$ \\
\hline Uemura et al. 2014 & \begin{tabular}{l} 
hemangioma of the forehead (1) \\
\hline
\end{tabular} & $2 \mathrm{y}$ \\
\hline
\end{tabular}

tumours which do not involve the anterior frontal plate ${ }^{(19)}$. Although the literature referring to the use of calvarial grafts in craniofacial surgery is limited, in most cases the results are rewarding (Table 2). Hendus and collegues emphasised the reconstruction of craniofacial bony frame in 15 patients with split calvarial bone grafts. The defects were caused by benign tumours [2], malignancies [3], traumas [6], and osteomyelitis [4]. In 12 of the cases, the entire anterior frontal sinus wall was affected. In the follow-up period between 2 and 8 years, the grafts remained stable in shape and size. There was no resorption, osteomyelitis, rejection, or donor site morbidity ${ }^{(20)}$. Liu et al. described a case of a 12-year-old girl with an aneurysmal bone cyst of the frontal sinus. Following bifrontal craniotomy and obliteration of the frontal sinus and nasofrontal duct, immediate reconstruction with split calvarial bone graft was performed. At one-year follow-up there was no recurrence, and the aesthetic outcome was favourable ${ }^{(21)}$. Uemura and colleagues reported a case of a 34-year-old woman with a hemangioma of the forehead. Following resection, reconstruction was performed with the outer table of split calvarial bone, harvested from the frontoparietal area. The postoperative course was uneventful, without any recurrence at 2-month follow-up ${ }^{(22)}$. Lubbers et al. reported a case of an 8-year old boy with a frontal and temporal bone osteoblastoma. Resection of the tumour through a coronal approach, and immediate reconstruction with an autologous calvarial split graft under navigation, achieved an accurate result without any need for a secondary operation ${ }^{(23)}$.
The importance of calvarial bone grafting for the repair of frontal sinus, orbital floor and nasal region was demonstrated by Maves and colleagues. Compared to autologous bone grafts harvested from the rib and the pelvis, the advantages of calvarial bone grafts were higher survival and using the same operative field ${ }^{(24)}$. Chang et al. described 3 cases of patients with symptomatic frontal sinus osteomas, who were operated by craniofacial approach. A coronal incision aided surgical exposure and the reconstruction with a calvarial bone graft in one patient. The outcome was favourable, and the patient presented no recurrence 3.5 years later ${ }^{(25)}$. Gil et al. outlined the use of split calvarial bone graft, following resection of tumours that involved frontal, nasal or orbital bones. In cases of benign tumours confined to the frontal sinus, type A osteotomy was performed. The anterior frontal sinus wall and the nasal frame are removed in one block, and obliteration of the frontal sinus with abdominal fat is usually performed, leaving the posterior frontal sinus wall intact. In cases of malignant tumours, type B osteotomy with a frontal sinus cranialisation was the treatment of choice. Split calvarial bone graft was required in 10 cases of craniofacial reconstruction ${ }^{(26)}$.

\section{Conclusion}

Our results concur with those reported above. In conclusion, many different grafts have been used in craniofacial reconstruction. According to our experience, autogenous calvarial bone graft seems to be one of the most suitable materials to use in the reconstruction of the frontal region, as it provides an optimal natural aesthetic outcome, while presenting minimal morbidity for the patient and the donor site. Furthermore, the availability of the graft makes it cost-effective, and harvesting within the same operative field decreases patient discomfort remarkably.

\section{Acknowledgement}

None

\section{Authorship contribution}

JC: Conception and design, Collection of data; SD, PDK: Data interpretation; JC, SD, PDK: Manuscript writing and final approval.

\section{Conflict of interest}

The authors do not have any conflicts of interest to declare.

\section{References}

1. Phillips, J.H., Rahn, B.A. Fixation effects on membranous and endochondral onlay bone-graft resorption. Plast Reconstr Surg 1988; 82:872-877.

2. König F. Der knöcherne Ersatz grosser Schädeldefekte. Zentralbl.1890. Chir 17 : 497.
3. Müller W. Zur Frage der temporären Schädelresektion anstelle der Trepanation. Zentralbl.1890. Chir 17: 65.

4. John L. Frodel. Jr. Calvarial Bone Graft Harvesting Techniques: Considerations for Their Use with Rigid Fixation Techniques in the Craniomaxillofacial Region. In: Greenberg Alex M. Joachim Prein Eds.
Craniomaxillofacial Reconstructive and Corrective Bone Surgery. Principles of Internal Fixation Using AO/ASIF Technique. Springer, 2002;700-712

5. Maas CS, Merwin GE, Wilson J, Frey MD, Maves MD. Comparison of biomaterials for facial bone augmentation. Arch Otolaryngol Head Neck Surg. 1990 May;116 
(5) : 551-556

6. Weber R, Draf W, Keerl R, Constantinidis Current aspects of frontal sinus surgery. II: External frontal sinus operation - osteoplastic approach. HNO. 1995 June; 43(6):358363.

7. Bockmühl U. Osteoplastic Frontal Sinusotomy and Reconstruction of Frontal Defects. In: Kountakis S, Senior B, Draf W. eds. The Frontal Sinus. Germany: Springer, 2005; 281-288.

8. Mohr C, Seifert V, Schettler D .Osteoplasty of osseous defects of the frontal bone and orbital roof - Indications, technique and results. Fortschr Kiefer GeSichtschir, 1994 $39: 43-46$.

9. Friedman $C D$, Costantino PD, Synderman $\mathrm{CH}$, Chow LC, Takagi S. Reconstruction of the frontal sinus and frontofacial skeleton with hydroxyapatite cement. Arch Facial Plast Surg. 2000 Apr-Jun;2(2):124-9.

10. Schmitz JP, Hollinger JO, Milam SB. Reconstruction of bone using calcium phosphate bone cements: a critical review. J Oral Maxillofac Surg. 1999 Sep;57(9):11226.

11. Goodrich JT, Argamaso R, Hall CD. Splitthickness bone grafts in complex craniofacial reconstructions. Pediatr Neurosurg. 1992;18(4):195-201.

12. Elisevich K, Bite U. En block forehead reconstruction with split-thickness cranial bone. Surg Neurol. 1991;35:384-388,

13. Sahoo N, Roy ID, Desai AP, Gupta V. Comparative evaluation of autogenous calvarial bone graft and alloplastic materials for secondary reconstruction of cranial defects. J Craniofac Surg. 2010 Jan;21(1):7982
14. Priya Jeyaraj. Efficacy and Versatility of the 3-D Titanium Mesh Implant in the Closure of Large Post-Craniectomy Osseous Defects, and its Therapeutic Role in Reversing the Syndrome of the Trephined: Clinical Study of a Case Series and Review of Literature. J Maxillofac Oral Surg. 2016 Mar; 15(1): 82-92s.

15. Cho HR, Roh TS, Shim KW, Kim YO, Lew DH, Yun IS., In Sik Yun. Skull Reconstruction with Custom Made Three-Dimensional Titanium Implant. Arch Craniofac Surg Vol.16 No.1 11-16.

16. Cannella DM, Hopkins LN. Superior sagittal sinus laceration complicating an autogenous calvarial bone graft harvest: report of a case. J Oral Maxillofac Surg. 1990 Jul;48(7):741-3.

17. Powell NB, Riley RW. Cranial bone grafting in facial aesthetic and reconstructive contouring. Arch Otolaryngol Head Neck Surg. 1987 Jul;113(7):713-9.

18. D. Marchac, A. Greensmith. Long-term experience with methylmethacrylate cranioplasty in craniofacial surgery. J Plast Reconstr Aesthet Surg. 2008 Jul;61(7):74452.

19. Draf W. Endonasal Frontal Sinus Drainage Type I-III According to Draf. In: Kountakis S, Senior B, Draf W. eds. The Frontal Sinus. Germany: Springer, 2005;223-226.

20. Hendus J, Draf W, Bockmühl U. Reconstruction of the frontoorbital frame using split-thickness calvarial bone grafts. Laryngorhinootologie. 2005 Dec;84(12):899904.

21. Liu JH, Newcomer MT, Murray AD, Myer CM 3rd. Aneurysmal bone cyst of the frontal sinus. Am J Otolaryngol. 2001 Jul-
Aug;22(4):291-3.

22. Uemura T, Sawai K, Kikuchi M, Masuoka J, Matsushima T. Technical strategies for en bloc resection and immediate reconstruction in hemangioma of the frontal bone. $J$ Craniofac Surg. 2014 Jul;25(4):e402-3.

23. Lübbers $H T$, Jacobsen $C$, Könü $D$, Matthews F, Grätz KW, Obwegeser JA. Surgical navigation in cranio-maxillofacial surgery: an evaluation on a child with a cranio-facioorbital tumour. Br J Oral Maxillofac Surg. 2011 Oct;49(7):532-7.

24. Maves MD, Matt BH. Calvarial bone grafting of facial defects. Otolaryngol Head Neck Surg. 1986 Nov;95(4):464-70

25. Chang SC, Chen PK, Chen YR, Chang CN. Treatment of frontal sinus osteoma using a craniofacial approach. Ann Plast Surg. 1997 May;38(5):455-9.

26. Gil Z, Abergel A, Leider-Trejo L et al. A comprehensive algorithm for anterior skull base reconstruction after oncological resections. Skull Base. 2007 Feb;17(1):25-37.

Dr. Petros D. Karkos

Department of Otolaryngology-Head

and Neck Surgery

AHEPA Hospital

Kiriakidi 1

Thessaloniki

54636, Greece

Tel: +302313303467

E-mail: pkarkos@aol.com 